\title{
Protection of aortic endothelial function in both normal and diabetic rats by intravenous bolus injection of a medium-chain triglyceride: fish oil emulsion
}

\author{
DAVID FONTAINE ${ }^{1}$, ANNE OTTO $^{1}$, LAURENCE PORTOIS $^{2}$, JEANINE FONTAINE $^{1}$, \\ GUY BERKENBOOM ${ }^{1}$, WILLY J. MALAISSE ${ }^{2}$ and YVON A. CARPENTIER ${ }^{2}$ \\ Laboratories of ${ }^{1}$ Physiology and Pharmacology and ${ }^{2}$ Experimental Surgery, \\ Brussels Free University, B-1070 Brussels, Belgium
}

Received April 26, 2006; Accepted July 7, 2006

\begin{abstract}
The bolus intravenous injection of a novel mediumchain triglyceride:fish oil emulsion (MCT:FO) was recently proposed as a tool to provoke a rapid and sustained increase of cell phospholipid content in long-chain polyunsaturated $\omega 3$ fatty acids, for instance in selected subjects prior to anesthesia and surgery. In this study, therefore, the possible protective effect of MCT:FO upon aortic endothelial function was investigated in both normal and diabetic rats. The animals were injected intravenously $20 \mathrm{~h}$ before sacrifice with $1.0 \mathrm{ml}$ of either saline, MCT:FO or a control medium-chain triglyceride:long-chain triglyceride emulsion. The vasomotor response of isolated aortic rings was then explored by assessing the relaxation provoked by increasing concentrations of acetylcholine in rings contracted with phenylephrine. Such measurements were performed before and after exposure of the aortic rings to suitable concentrations of oxidized LDL. In both normal and diabetic rats, the prior injection of the MCT:FO emulsion protected the aortic rings against the deleterious effect of oxidized LDL. In the diabetic rats, a beneficial effect of the MCT:FO emulsion was even observed prior to exposure of the aortic rings to oxidized LDL. These findings support the view that this novel procedure is indeed appropriate to protect endothelial function against oxidative stress.
\end{abstract}

\section{Introduction}

We have recently reported that the bolus intravenous injection of a novel medium-chain triglyceride-fish oil emulsion $(8: 2$,

Correspondence to: Professor Willy J. Malaisse, Laboratory of Experimental Surgery, Brussels Free University, 40 Avenue J. Wybran, B-1070 Brussels, Belgium

E-mail: malaisse@ulb.ac.be

Key words: medium-chain triglyceride:fish oil emulsion, aortic rings, endothelial function, streptozotocin-induced diabetic rats
MCT:FO) to both rats and human subjects increases within $60 \mathrm{~min}$ and for at least $24-48 \mathrm{~h}$ the phospholipid content in long-chain polyunsaturated $\omega 3$ fatty acids $(\omega 3)$ in circulating leucocytes or platelets (1) and even in extravascular cells such as hepatocytes (2) or skeletal myocytes (3). It was considered, therefore, that such a procedure may rapidly restore a sufficient amount of $\omega 3$ in cell phospholipids and, for instance, protect selected patients against the risk of cardiac arrhythmia when undergoing anesthesia and/or surgery (1).

As a first step towards assessing the validity of the latter proposal, the present study aimed mainly at investigating the possible protective effects of the intravenous injection of the MCT:FO emulsion to both normal and streptozotocin-induced diabetic rats against the deleterious action of oxidized lowdensity lipoproteins on the vasomotor behaviour of isolated aortic rings.

\section{Materials and methods}

Emulsions. The MCT:FO emulsion consists of a $20 \%(\mathrm{w} / \mathrm{v})$ mixture of $80 \%$ medium-chain triacylglycerols and $20 \%$ fish oil. It also contains $2.5 \%(\mathrm{w} / \mathrm{v})$ glycerol, $1.2 \%(\mathrm{w} / \mathrm{v}) \mathrm{egg}-$ derived phospholipids as emulsifier and $0.4 \%(\mathrm{w} / \mathrm{v})$ all-rac- $\alpha-$ tocopherol. The control MCT:LCT emulsion (Medialipid ${ }^{\circledR}$, B. Braun), currently used for parenteral nutrition consists of a $20 \%(w / v)$ mixture of equal amounts of MCT lipids and soybean LCT (LCT emulsion).

Animals and treatments. All animals procedures were approved by our university ethics committee. Adult male Wistar rats were housed in a controlled environment and given free access to standard rat chow and tap water. They weighed between $300-350 \mathrm{~g}$ on the day of sacrifice. When 8 weeks old, some rats received a single i.p. injection of streptozotocin (STZ $75 \mathrm{mg} /$ $\mathrm{kg}$, prepared in saline and citrate buffer $\mathrm{pH} 4.5$ ). After a 5week period, with twice a week blood glucose level monitoring using an accutrend sensor (Roche Diagnostics), these rats were used in the experimental setting.

Pilot experiments conducted in normal rats aimed at optimizing the experimental setting, with emphasis on the concentration-response relationship for the effects of oxidized LDL on the vasomotor responses. 
In further experiments, three groups of rats were usually examined. In the first group, the animals received intravenously $1.0 \mathrm{ml}$ of the MCT:FO emulsion by catheterization of the saphenous vein under chloral hydrate $(400 \mathrm{mg} / \mathrm{kg})$ anesthesia. In the second group, the rats were injected with $1.0 \mathrm{ml}$ of the LCT emulsion. The third group served as control, the rats receiving an isotonic saline injection. All animals were treated with a single dose of carprofen $(4.0 \mu \mathrm{g} / \mathrm{g} \mathrm{s.c.;}$ Rimadyl ${ }^{\circledR}$, Pfizer, NY, USA) $3 \mathrm{~h}$ before the anesthesia. After the intravenous administrations, the rats were housed for a $20-\mathrm{h}$ period and then eventually sacrificed under carbon dioxide. Their thoracic aorta was removed, cleaned of adhering fat and cut into rings of 4-mm length. During the dissection, utmost care was taken to protect the endothelial lining.

Preparation of $L D L$. LDL fraction was isolated from human plasma and collected in $1 \mathrm{mM}$ EDTA by sequential ultracentrifugation, with density adjustments by potassium bromide (4), and oxidized on $\mathrm{CuSO}_{4}$ for $24 \mathrm{~h}$ as previously described (5). The final total concentration of LDL used in the bath was $3.0 \mathrm{mg} / 1$, different oxidation levels being achieved by variations in the proportion of oxidized LDL to total LDL. Pilot experiments were performed using a standardized solution of oxidized LDL from commercial kits (Mercodia ${ }^{\mathrm{TM}}$ ).

Studies of vasomotor responses. All rings were mounted under $2 \mathrm{~g}$ resting tension on stainless steel hooks in $20 \mathrm{ml}$ organ baths. These organ chambers were filled with Krebs-Henseleit solution of the following composition (in $\mathrm{mM}$ ): $\mathrm{NaCl} 118.1$, $\mathrm{KCl} 4.7, \mathrm{MgSO}_{4} 1.2, \mathrm{KH}_{2} \mathrm{PO}_{4} 1.2, \mathrm{CaCl}_{2} 2.5, \mathrm{NaHCO}_{3} 25$, and D-glucose 5, equilibrated with a mixture of $95 \% \mathrm{O}_{2} / 5 \% \mathrm{CO}_{2}$ and kept at $37^{\circ} \mathrm{C}$. Tension was measured isometrically with a force transducer (Grass FTO3C; Grass Instruments, Quincy, MA, USA) and continuously recorded with a transducer amplifier (Janssen Scientific Instruments) and a pen recorder. After 60 min equilibration, the rings were stretched progressively until the optimal point of the length-tension relation was reached, as previously described $(5,6)$. A concentrationresponse curve to acetylcholine (Ach, $10^{-8}$ to $10^{-4} \mathrm{M}$ final concentration in the bath) was first constructed on phenylephrine $\left(10^{-5}\right.$ to $\left.10^{-6} \mathrm{M}\right)$ preconstricted aortas in the absence of oxidized LDL in order to assess the vascular reactivity of each ring. This concentration response curve will be called below the first relaxation test. After a washing out period of $30 \mathrm{~min}$ while the tension in the rings returns to baseline, oxidized LDL were added to the bath for a 30-min incubation period and a second relaxation test was performed with LDL left in the bath.

To exclude an altered sensitivity of the preparations to acetylcholine, 3 preparations were studied in parallel; one served as control, and 2 concentration-response curves were constructed at 1-h intervals. As there was no tachyphylaxis to Ach, which is in agreement with our previous studies $(5,6)$, the responses in the absence or in the presence of LDL were compared in adjacent preparations (using mainly the second concentration-response curves).

To prevent foaming with LDL, Antifoam B (1 volume \%) was added to each organ bath. As pointed out in our previous study $(5,7)$, this antifoam did not alter the physical integrity of LDL and did not modify the responses to various agents tested.

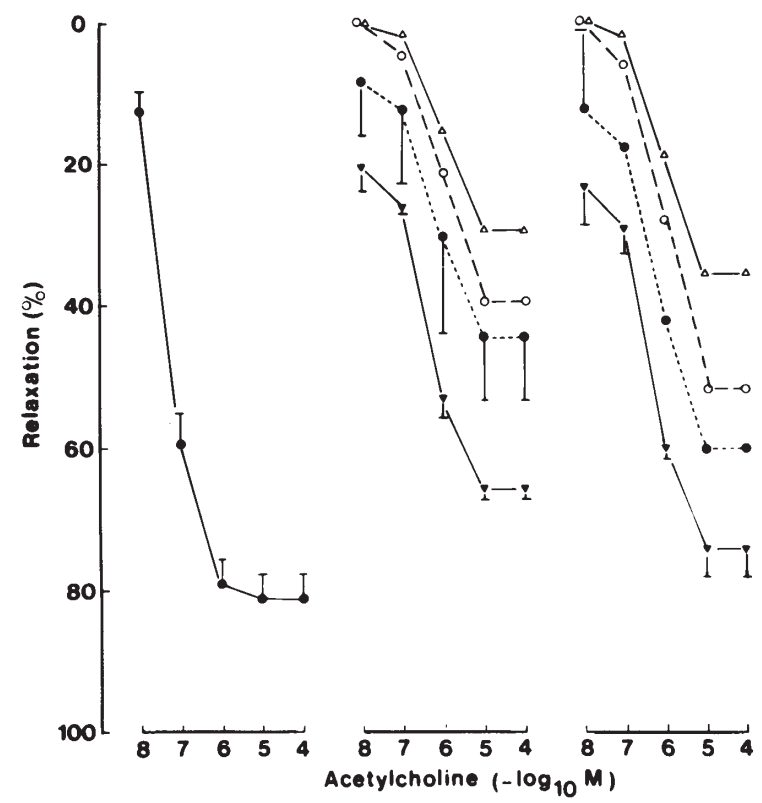

Figure 1. First relaxation test (left panel) and second relaxation test (middle and right panels) in aortic rings from normal rats exposed, after the first test, to increasing concentrations of oxidized LDL ( $20 \mu \mathrm{U} / 1$ : closed triangles and solid line; $40 \mu \mathrm{U} / \mathrm{l}$ : closed circles and dotted line; $80 \mu \mathrm{U} / \mathrm{l}$ : open circles and dashed line; $160 \mu \mathrm{U} / 1$ : open triangles and solid line). Mean values $( \pm$ SEM) are expressed as the percent relaxation relative to either the reference value found in the sole presence of phenylephrine (left and middle panels) or the paired maximal relaxation caused by phenylephrine in the same ring during the first test (right panel), and refer to 6 (left panel) or 1-2 (middle and right panels) rings.

All experiments were performed in the absence of cyclooxygenase inhibitor, because indomethacin does not affect endothelium-dependent relaxations in rat aorta $(4,6)$.

Presentation of results and statistical analysis. All results are expressed as mean $( \pm$ SEM) together with the number of separate determinations (n) or degree of freedom (d.f.). The relaxations are expressed in $\mathrm{cm}$ on the pen recorder, corresponding to $1.0 \mathrm{~g}$ for $4 \mathrm{~cm}$. Statistical analysis was performed using Student's t-test.

\section{Results}

Response to oxidized LDL. Fig. 1 summarizes the results of a first set of experiments designed to assess the effect of oxidized LDL (from Mercodia) on the response to phenylephrine and acetylcholine in aortic rings obtained from a normal rat. A first relaxation test documented the effect of cumulative doses of acetylcholine (final concentration in the baths: $10^{-8}$ to $10^{-4} \mathrm{M}$ ) on phenylephrine pre-constricted rings $\left(10^{-5} \mathrm{M}\right)$. As illustrated in Fig. 1 (left panel), the tension decreased, as a function of the concentration of acetylcholine from a control value of $5.70 \pm$ to $0.62 \mathrm{~cm}$ to a minimal value of $1.12 \pm 0.23 \mathrm{~cm}(\mathrm{n}=6$ in both cases), suggesting a functional endothelium. The aortic rings were then exposed for $30 \mathrm{~min}$ to increasing concentrations of oxidized LDL (20-160 $\mu \mathrm{U} /$ liter final concentration). Under these conditions, the relaxing effect of acetylcholine, as assessed during a second test, was obviously impaired (Fig. 1, middle panel). Essentially comparable information was obtained, when the relaxation found after 


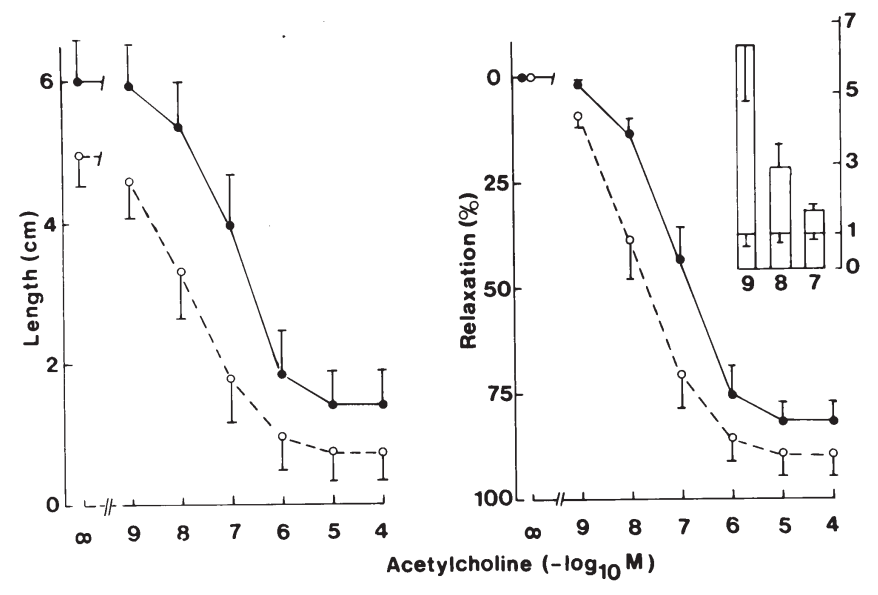

Figure 2. First relaxation test in aortic rings from 2 groups of 3 normal rats ( $n=3$ in each group). Mean value $( \pm$ SEM) in group A (open circles and dashed line) and group B (closed circles and solid line) are expressed either in absolute terms (left panel) or as a percent relaxation (right panel), and are derived from 13-14 rings. The inset refers to the fractional relaxation found in the rats of group A relative to the mean value found at the same concentration of acetylcholine in the rats of group B.

exposure to oxidized LDL was expressed relative to the paired maximal relaxation recorded in the same aortic ring before exposure to oxidized LDL (Fig. 1, right panel). Incidentally, the absolute value for the tension caused by phenylephrine in the absence of acetylcholine during the second test, when expressed relative to the paired value found in the same aortic ring during the first test, was inversely related to the concentration of oxidized LDL, representing after exposure to $80-160 \mu \mathrm{U} / 1$ and $40 \mu \mathrm{U} / 1$ respectively $60.7 \pm 0.5 \%$ and $80.2 \pm 9.2 \%$ of the mean value found after exposure to only $20 \mu \mathrm{U} / 1$, i.e. $100.0 \pm 8.5 \%(\mathrm{n}=2$ in all cases). Such a situation was confirmed in further experiments (see below).

In light of these findings, a further series of experiments was conducted in aortic rings obtained from two groups of three rats each (group A and B) injected with distinct but unidentified emulsions. As illustrated in Fig. 2, the mean tension caused, in the absence of acetylcholine, by phenylephrine $\left(10^{-6} \mathrm{M}\right)$ during the first relaxation test was lower $(\mathrm{p}<0.06)$ in group A than in group B. The relative extent of the relaxation caused by acetylcholine was higher, however, in group A than in group B.

After the first relaxation test, the aortic rings were exposed for $30 \mathrm{~min}$ to a fixed total concentration of LDL $(3.0 \mathrm{mg} / \mathrm{ml})$, prepared from normal human subjects, with increasing ratios $(0 / 1,1 / 16,1 / 4$ and $1 / 1)$ of oxidized/total LDL. As shown in Fig. 3 (left panel), the second relaxation test conducted in rings not exposed to oxidized LDL although involving much smaller numbers of aortic rings ( $\mathrm{n}=4$ and 5) provided essentially the same results as those illustrated in Fig. 2. The exposure of the aortic rings to increasing concentrations of oxidized LDL resulted in a concentration-related impairment of aortic contractility. First, as already observed in the first set of experiments, the maximal tension caused by phenylephrine (in the absence of acetylcholine) during the second test, was decreased after exposure to a high concentration of oxidized LDL (data not shown). Second, the relative magnitude of the relaxing action of acetylcholine was also inversely related to the concentration of oxidized LDL. The middle panel of Fig. 3
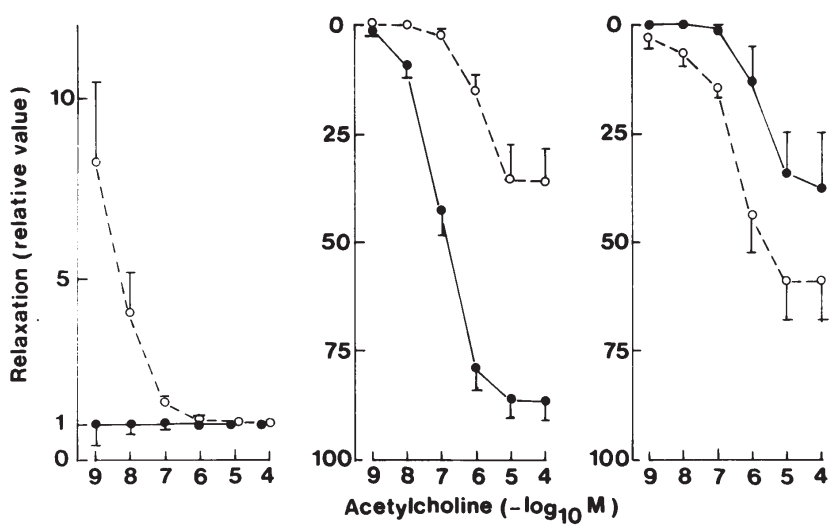

Figure 3. Second relaxation test in aortic rings from normal rats $(n=3$ in each group). Left panel: comparison between rings obtained from rats in group A (open circles and dotted line) and group B (closed circles and sold line) and not exposed to oxidized LDL, the results being expressed relative to the mean value obtained at each concentration of acetylcholine in the latter animals. Middle panel: comparison between rings obtained from rats in group B and preincubated in the absence (closed circles and solid line) or presence of oxidized LDL (1/4 oxidized/total LDL ratio). Right panel: comparison between rings obtained from rats in group A (open circles and dotted line) or group B (closed circles and solid line) and preincubated in the presence of oxidized LDL (1/16 oxidized/total LDL ratio). Mean values ( \pm SEM) refer to $4-5$ (left panel) or 3 (middle and right panels) rings.

illustrates the results obtained in aortic rings from rats in group B, incubated for $30 \mathrm{~min}$ in the absence or presence of oxidized LDL (1/4 oxidized/total LDL ratio). This second effect of oxidized LDL was not identical in group A and B. As illustrated in Fig. 3 (right panel), which refers to aortic rings exposed for $30 \mathrm{~min}$ to a low concentration of oxidized LDL (1/16 oxidized/total ratio), the difference between group $\mathrm{A}$ and $\mathrm{B}$, in terms of the relative extent of the relaxation caused by acetylcholine averaged, in the $10^{-7}$ to $10^{-4} \mathrm{M}$ range, $22.8 \pm 6.0 \%$ (d.f.=16; p <0.005) of the maximal tension recorded in the absence of the cholinergic agent. Taken as a whole, these findings emphasize the need of comparing in aortic rings obtained from the same animals the response to phenylephrine and acetylcholine before and after exposure to oxidized LDL.

Normal rats. Aortic rings were obtained from five rats injected with the LCT emulsion and six rats injected with the MCT:FO emulsion. During a first relaxation test, neither the absolute value for the tension caused by phenylephrine $\left(10^{-5} \mathrm{M}\right)$ nor the relative extent of the relaxation caused by acetylcholine (also $\left.10^{-5} \mathrm{M}\right)$ were significantly different $(\mathrm{p} \geq 0.4)$ in the two groups of rats, with overall mean respective values of $4.62 \pm 0.32 \mathrm{~cm}$ and $79.1 \pm 2.3 \%(\mathrm{n}=29$ in both cases $)$.

The aortic rings were then incubated for $30 \mathrm{~min}$ in the absence or presence of oxidized LDL (1/16 oxidized/total LDL ratio). In the aortic rings obtained from the animals injected with the LCT emulsion and exposed to oxidized LDL, the maximal tension recorded during the second relaxation test in the sole presence of phenylephrine was lower $(n=8$; $\mathrm{p}<0.03$ ) than the paired value found at the same time in aortic rings obtained from the same rats but not exposed to oxidized LDL. Such was not the case, however, in the aortic rings from rats injected with the MCT:FO emulsion $(n=8)$. Such paired ratios averaged, in the rats injected with the LCT emulsion, 
Table I. Relaxation by acetylcholine of aortic rings obtained from rats injected with either an LCT or MCT:FO emulsion and incubated in the absence or presence of oxidized LDL before exposure to phenylephrine $\left(10^{-6} \mathrm{M}\right)$.

\begin{tabular}{|c|c|c|c|c|c|}
\hline \multirow{2}{*}{\multicolumn{2}{|c|}{$\begin{array}{l}\text { Emulsion } \\
\text { Oxidized LDL }\end{array}$}} & \multicolumn{2}{|c|}{ LCT } & \multicolumn{2}{|c|}{ MCT:FO } \\
\hline & & Nil & $40 \mu \mathrm{U} / 1$ & Nil & $40 \mu \mathrm{U} / \mathrm{l}$ \\
\hline \multirow[t]{5}{*}{ Acetylcholine } & $10^{-8} \mathrm{M}$ & $27.6 \pm 6.5(6)^{a}$ & $10.3 \pm 2.3(8)$ & $32.0 \pm 7.7(5)$ & $17.7 \pm 2.9(10)$ \\
\hline & $10^{-7} \mathrm{M}$ & $51.9 \pm 10.5(5)$ & $17.8 \pm 4.3(8)$ & $57.0 \pm 14.5(5)$ & $31.6 \pm 5.5(10)$ \\
\hline & $10^{-6} \mathrm{M}$ & $88.2 \pm 12.2(5)$ & $52.4 \pm 9.2(8)$ & $91.0 \pm 14.9(5)$ & $71.9 \pm 8.4(10)$ \\
\hline & $10^{-5} \mathrm{M}$ & $104.0 \pm 15.7(5)$ & $71.6 \pm 11.3(8)$ & $104.2 \pm 15.9(5)$ & $94.5 \pm 10.2(10)$ \\
\hline & $10^{-4} \mathrm{M}$ & $104.6 \pm 15.3(5)$ & $72.0 \pm 11.1(8)$ & $104.2 \pm 15.9(5)$ & $94.8 \pm 10.2(10)$ \\
\hline
\end{tabular}

${ }^{a}$ The results are expressed relative to the percent relaxation caused by $10^{-5} \mathrm{M}$ acetylcholine in the same aortic ring during a first relaxation test

$76.0 \pm 3.6 \%(n=8 ; \mathrm{p}<0.005)$ of the mean corresponding value observed in the rats injected with the MCT:FO emulsion $(100.0 \pm 5.3 \% ; \mathrm{n}=8)$.

In the rats injected with the control LCT emulsion, the response to acetylcholine during the second relaxation test was also severely impaired in the aortic rings exposed to oxidized LDL. For reasons already considered, the relative extent of the relaxing action of acetylcholine during the second test was expressed relative to the paired maximal relaxing action of the cholinergic agent as recorded, in the same ring, when exposed to $10^{-5} \mathrm{M}$ acetylcholine during the first test. The decrease in acetylcholine relaxing action attributable to oxidized LDL in the rings from rats injected with the LCT emulsion only achieved statistical significance $(\mathrm{p}<0.05$ to $\mathrm{p}<0.005$; d.f. $=11$ ) at $10^{-8}, 10^{-7}$ and $10^{-6} \mathrm{M}$, when judged by the data listed in Table I. However, when compared within each animal, the relaxing action of acetylcholine $\left(10^{-5}\right.$ and $\left.10^{-4} \mathrm{M}\right)$ remained significantly lower $(\mathrm{p}<0.02)$ in the rings exposed to oxidized LDL than in the control rings not exposed to oxidized LDL. For instance, at $10^{-4} \mathrm{M}$ acetylcholine, the results recorded in the aortic rings exposed to oxidized LDL averaged $76.0 \pm 7.8 \%(n=8 ; p<0.02)$ of the paired control value (no oxidized LDL) found in the same animal(s).

The MCT:FO emulsion also protected against the effect of the oxidized LDL upon the response to acetylcholine. The mean relative extent of the relaxing action of acetylcholine, as listed in Table I, was always higher, at all concentrations of the cholinergic agent $\left(10^{-8}\right.$ to $\left.10^{-4} \mathrm{M}\right)$, in the aortic rings obtained from rats injected with the MCT:FO emulsion and exposed to oxidized LDL than in the rings from rats injected with the LCT emulsion and also exposed to oxidized LDL. In order to assess specifically the protective action of the MCT:FO emulsion against the deleterious effect of oxidized LDL, the relaxing action of acetylcholine in rings exposed to oxidized LDL (Table I) were expressed relative to the mean corresponding value found in rings from the same type of rats not preincubated with oxidized LDL and eventually exposed to the same concentration of the cholinergic agent (Table I). Under these conditions, the results obtained in rats injected with the MCT:FO emulsion were always higher than those recorded in the rats injected with the LCT emulsion (Fig. 4). The difference between the two sets of results was not significantly affected $(\mathrm{p}>0.8)$ by the concentration of

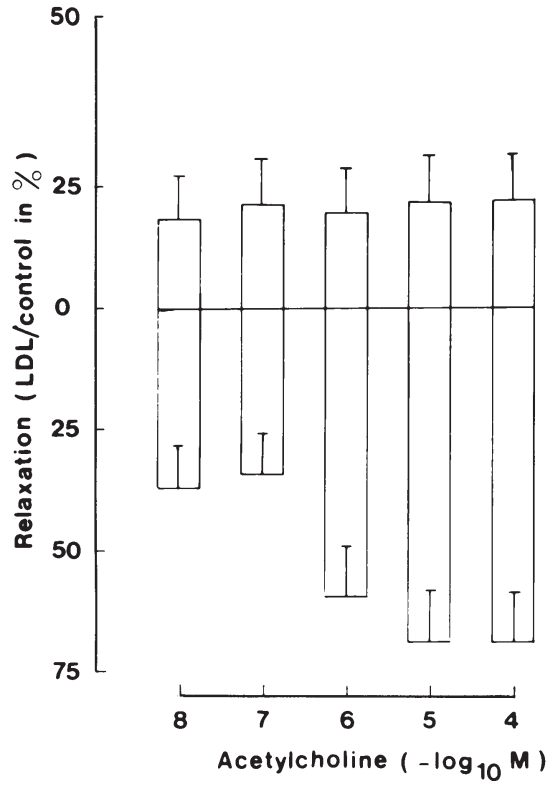

Figure 4. Relaxing action of acetylcholine $\left(10^{-8}\right.$ to $\left.10^{-4} \mathrm{M}\right)$ in aortic rings obtained from rats injected with an LCT or MCT:FO emulsion (5-6 rats in each group). The results obtained in rings exposed to oxidized LDL ( $40 \mu \mathrm{U} / \mathrm{l})$ are expressed relative to the mean corresponding values found in control rings not exposed to LDL (see Table I for primary data). The data collected in the rats injected with the LCT emulsion are illustrated below the 'zero' horizontal line. The data recorded in rats injected with the MCT:FO emulsion are illustrated above the 'zero' horizontal line, after subtraction of the results obtained in the rats injected with the LCL emulsion. Mean values ( \pm SEM) refer to 8 (LCT rats) and 10 (MCT:FO rats) rings.

acetylcholine, with an overall mean value of $20.5 \pm 6.0 \%$ (d.f. $=80 ; p<0.001)$. The results illustrated in Fig. 4 also document that, as expected from prior results, the deleterious effect of oxidized LDL was, in relative terms, most marked at the lowest concentration(s) of acetylcholine.

These findings were confirmed in further experiments conducted in normal rats injected with either the MCT:FO or control LCT emulsion. During the first relaxation test, the tension caused by phenylephrine $\left(10^{-6} \mathrm{M}\right)$ was not significantly different $(\mathrm{p}>0.55)$ in the rats injected with the MCT:FO $(6.50 \pm 0.95 \mathrm{~cm} ; \mathrm{n}=9)$ or LCT $(7.03 \pm 0.36 \mathrm{~m} ; \mathrm{n}=10)$ emulsion. Likewise, the relative magnitude of the relaxing action of acetylcholine during this first test failed to differ significantly 


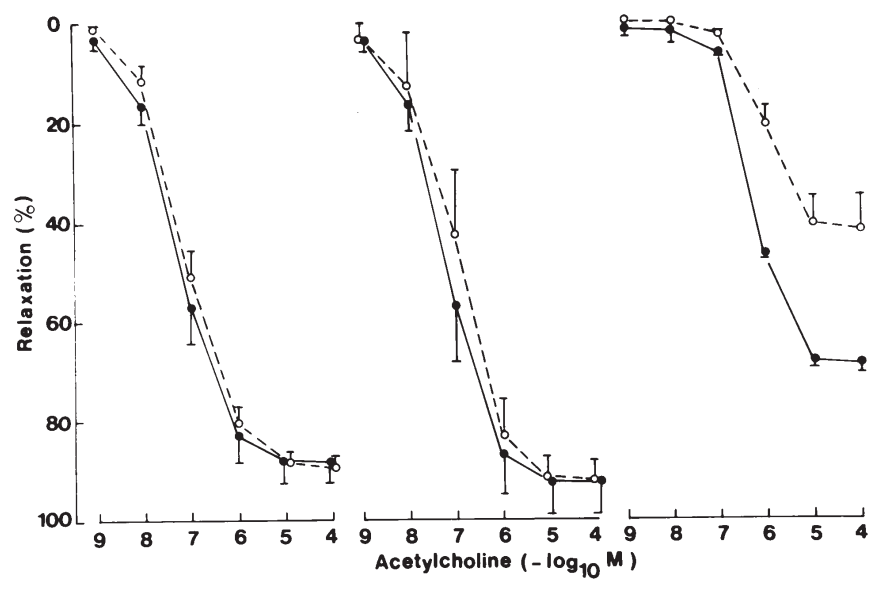

Figure 5. First relaxation test (left panel), second relaxation test in aortic rings not exposed to oxidized LDL (middle panel) and second relaxation test in aortic rings exposed to oxidized LDL (1/16 oxidized/total LDL ratio). The data were obtained from rats injected with either the MCT:FO emulsion (closed circles and solid line) or a control MCT emulsion (open circles and dotted line). Mean values ( \pm SEM) refer to 9-10 rings (left panel), 3-4 rings (middle panel) and 2 rings (right panel).

in the two groups of rats (Fig. 5, left panel). Such was also the case during the second relaxation test in aortic rings not exposed to oxidized LDL (Fig. 5, middle panel). In both cases, the trend was towards a greater relative relaxing action of acetylcholine in the rings obtained from rats injected with the MCT:FO, as distinct from LCT, emulsion.

Similar to previous experiments, the tension measured in the sole presence of phenylephrine $\left(10^{-6} \mathrm{M}\right)$ during the second relaxation test progressively decreased as the oxidized/total LDL ratio increased from $1 / 16$ to $1 / 1$, the values reached in the latter case representing $66.0 \pm 11.4 \%(n=4 ; p<0.06)$ of the paired value found in the same rat(s) in the former case.

The exposure to oxidized LDL also impaired the relaxing action of acetylcholine. For instance, in the aortic rings exposed to oxidized LDL at an oxidized/total ratio of 1/16, the maximal response to the cholinergic agent $\left(10^{-4} \mathrm{M}\right)$ was decreased from $92.7 \pm 6.4 \%(n=3)$ to $69.0 \pm 2.0 \%(n=2)$ in the rats injected with the MCT:FO emulsion and from $92.0 \pm 3.8 \%(n=4)$ to $42.0 \pm 7.0 \%(\mathrm{n}=2)$ in the animals injected with the control LCT emulsion. This alteration of the functional response to acetylcholine is further documented by comparison of the results illustrated in the middle and right panels of Fig. 5.

As documented in Fig. 5 (right panel), in the aortic rings exposed to the low concentration of oxidized LDL (oxidized/ total LDL ratio to $1 / 16$ ) the mean relative magnitude of the relaxing action of acetylcholine $\left(10^{-9}\right.$ to $\left.10^{-4} \mathrm{M}\right)$ was always higher in the rings obtained from rats injected with the MCT:FO emulsion than in those obtained from animals injected with the LCT emulsion. Such a difference achieved statistical significance $(\mathrm{p} \leq 0.05)$ in the $10^{-7}$ to $10^{-5} \mathrm{M}$ range of acetylcholine concentrations. Between $10^{-7}$ and $10^{-4} \mathrm{M}$ acetylcholine, the relative extent of the relaxing action of the cholinergic agent averaged in the rat injected with the LCT emulsion only $50.5 \pm 4.8 \%(n=8 ; p<0.001)$ of the corresponding mean value recorded at the same concentration of acetylcholine in the aortic rings from rats injected with the MCT:FO emulsion $(100.0 \pm 1.7 \% ; n=8)$. The protective effect of the MCT:FO emulsion faded out, however, at a higher

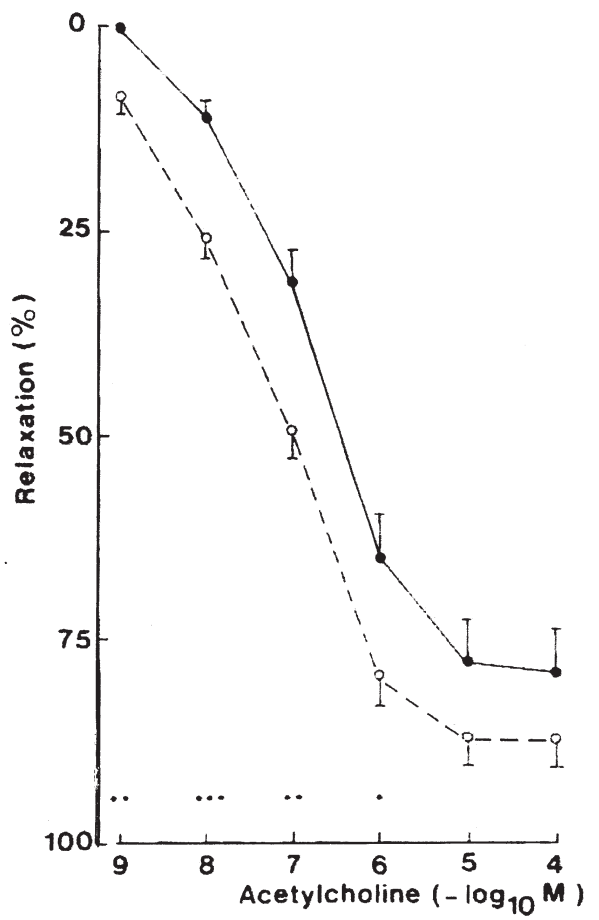

Figure 6. First relaxation test in aortic rings obtained from STZ rats injected with either the LCT (closed circles and solid line) or MCT:FO (open circles and dashed line) emulsion (4 rats in each group). Mean values ( \pm SEM) refer to 14-15 rings and are shown together with the statistical significance of differences between the two sets of data $\left({ }^{*} \mathrm{p}<0.025 ;{ }^{* *} \mathrm{p}<0.005 ;{ }^{* * *} \mathrm{p}<0.001\right)$.

concentration of oxidized LDL (oxidized/total LDL ratio of $1 / 4$ and 1/1). Thus, in this case, the relative magnitude of the relaxing action of acetylcholine $\left(10^{-7}\right.$ to $\left.10^{-4} \mathrm{M}\right)$ recorded in the aortic rings from rats injected with the MCT:FO emulsion averaged no more than $106.0 \pm 16.3 \%(n=16 ; p>0.7)$ of the corresponding value found at the same concentration of the cholinergic agent and within the same experiment in rings obtained from animals injected with the LCT emulsion.

Taken as a whole, the experiments conducted in normal rats injected with the MCT:FO emulsion thus indicate that it failed to affect significantly the functional response to phenylephrine and acetylcholine in aortic rings not exposed to oxidized LDL but exerted a significant protective action in rings exposed to a relatively low concentration of oxidized LDL.

STZ rats. The mean body weights of the STZ rats injected with either saline, the LCT emulsion or the MCT:FO emulsion were not significantly different from one another $(p \geq 0.3)$, with an overall mean value of $288 \pm 12 \mathrm{~g}(\mathrm{n}=25)$. Likewise, the mean glycemia was not significantly different $(p \geq 0.19)$ in the three groups of STZ rats, with an overall mean value of $16.44 \pm 1.70 \mathrm{mM}(\mathrm{n}=24)$.

During the first relaxation test conducted in aortic rings from STZ rats, the tension caused by phenylephrine $\left(10^{-5} \mathrm{M}\right)$ was not significantly different $(p>0.3)$ in the rats injected with the LCT emulsion $(6.65 \pm 0.37 \mathrm{~cm} ; \mathrm{n}=33)$ or MCT:FO emulsion $(6.09 \pm 0.47 \mathrm{~cm} ; \mathrm{n}=30)$. However, the mean relaxation caused by acetylcholine was, in the $10^{-9}$ to $10^{-4} \mathrm{M}$ range, higher in the latter than former rats. This is illustrated in Fig. 6, which refers to a representative set of experiments conducted in 4 rats 


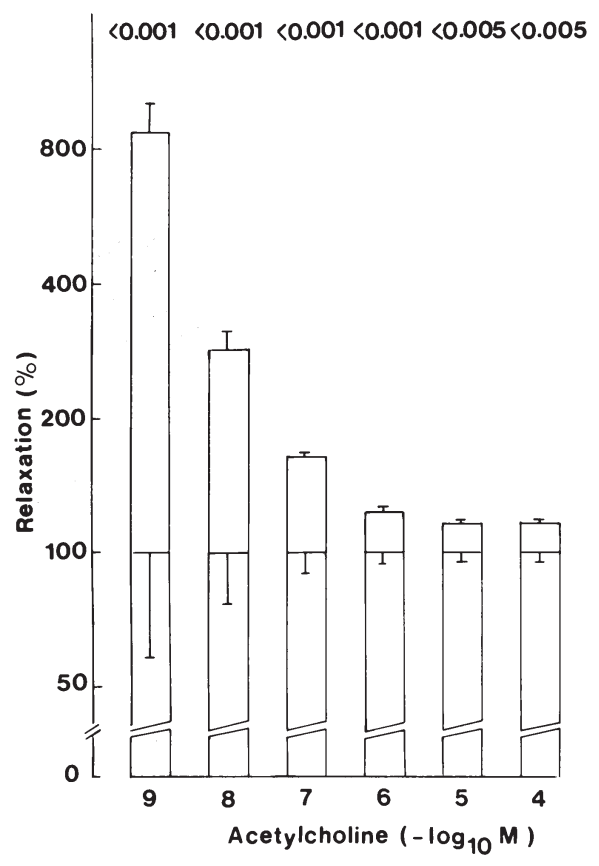

Figure 7. First relaxation test in aortic rings obtained from STZ rats injected with either the LCT or MCT:FO emulsion (8-9 rats in each group). The results obtained in the rats injected with the MCT:FO emulsion are expressed relative to the mean corresponding values recorded at the same concentration of acetylcholine in the rats injected with the LCT emulsion. Mean values ( \pm SEM) refer to $30-33$ rings, are ranged on a logarithmic scale, and are shown together with the statistical significance of differences between the two sets of data.

in each group. In the $10^{-8}$ to $10^{-6} \mathrm{M}$ range of acetylcholine concentrations, the shift of the concentration-response relationship in the rats injected with the LCT versus MCT:FO emulsion corresponded to a difference in concentration of the cholinergic agent close to one order of magnitude, e.g. from $10^{-8}$ to $10^{-7} \mathrm{M}$. These findings were confirmed in another set of comparable experiments conducted in 4-5 STZ rats injected with either the LCT or MCT:FO emulsion. The overall mean results are illustrated in Fig. 7. It indicates that, at all concentrations of acetylcholine $\left(10^{-9}\right.$ to $\left.10^{-4} \mathrm{M}\right)$, the relaxation was significantly higher $(\mathrm{p} \leq 0.005)$ in the rats injected with the MCT:FO emulsion than in those injected with the LCT emulsion. The former results represented between $8.67 \pm 1.91$ $\left(\mathrm{n}=30\right.$ at $10^{-9} \mathrm{M}$ acetylcholine $)$ and $1.16 \pm 0.03\left(\mathrm{n}=30\right.$ at $10^{-5}$ and $10^{-4} \mathrm{M}$ acetylcholine) times the corresponding mean values found in the rats injected with the LCT emulsion and derived in all cases from 33 measurements in individual aortic rings.

Although the normal and STZ rats were examined in two separate series of experiments, three sets of findings point towards an alteration or aortic endothelium function in the diabetic animals. First, whether in the rats injected with the LCT or MCT:FO emulsion, the tension caused by phenylephrine during the first relaxation test was higher in the STZ rats than in the normal animals. It averaged, in the former rats, $117.4 \pm 5.5 \%(n=63 ; \mathrm{p}<0.04)$ of the mean corresponding value found in normal rats injected with the same emulsion $(100.0 \pm 6.0 \% ; \mathrm{n}=48)$. Second, during the second test conducted in aortic rings not exposed to oxidized LDL, the relaxation caused by acetylcholine was higher in the normal rats than in

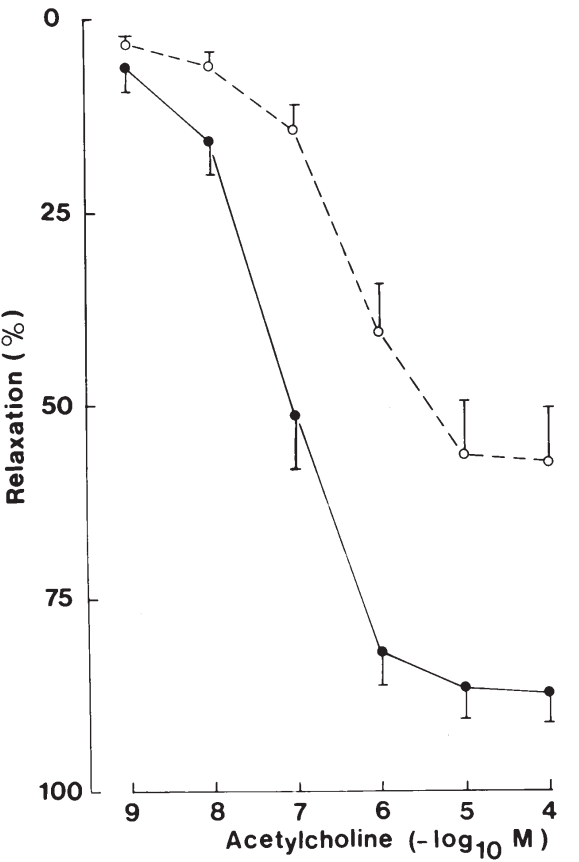

Figure 8. Second relaxation test in aortic rings obtained from 9 STZ rats injected with saline and preincubated for $30 \mathrm{~min}$ in the absence (closed circles and solid line) or presence (open circles and dashed line) of oxidized LDL (1/16 oxidized/total LDL ratio). Mean values ( \pm SEM) refer to 13-16 rings.

the diabetic animals. For instance, the fractional relaxation caused by $10^{-8} \mathrm{M}$ acetylcholine averaged in the STZ rats $58.2 \pm 13.0 \%(\mathrm{n}=25 ; \mathrm{p}<0.04)$ of the mean corresponding value found in normal rats injected with the same lipid emulsion $(100.0 \pm 13.5 \% ; n=35)$. Third, when comparing STZ rats injected with the LCT versus MCT:FO emulsion and at variance with the situation found in normal rats, the exposure of the aortic rings to oxidized LDL failed to affect in a significantly different manner the reference tension measured during the second relaxation test in the sole presence of phenylephrine. Such was the case, whether the results were considered in absolute terms or relative to the paired measurement made in the same ring during the first relaxation test. For instance, when the determinations made during the second relaxation test in aortic rings that had been exposed to oxidized LDL were expressed relative to the corresponding value(s) found in aortic ring(s) of the same animal not exposed to oxidized LDL, the readings made in STZ rats injected with the LCT emulsion averaged $89.6 \pm 4.7 \%(\mathrm{n}=20)$ of those made in STZ rats injected with the MCT:FO emulsion (100.0 $5.6 \%$; $\mathrm{n}=17)$. The former percentage not only failed to differ significantly ( $>00.1)$ from the latter one, but was also significantly higher $(\mathrm{p}<0.03)$ than that found in normal rats $(73.7 \pm 3.9 \%$; $\mathrm{n}=12)$.

Nevertheless, in aortic rings from STZ rats, like in those from normal rats, prior exposure to oxidized LDL (1/16 oxidized/total LDL ratio) altered the response to acetylcholine. For instance, Fig. 8 illustrates the results collected in STZ rats injected with saline $\sim 20 \mathrm{~h}$ before sacrifice. The mean relative extent of the relaxation provoked by acetylcholine $\left(10^{-9}\right.$ to $10^{-4} \mathrm{M}$ ) in the presence of $10^{-5} \mathrm{M}$ phenylephrine was always 


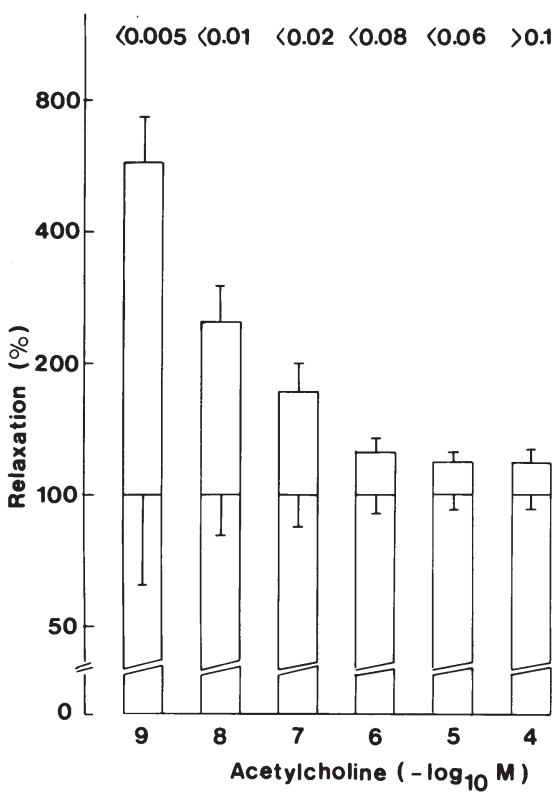

Figure 9. Second relaxation test in aortic rings obtained from STZ rats injected with either the LCT or MCT:FO emulsion (8-9 rats in each group). The results obtained in the rings from rats injected with the MCT:FO emulsion are expressed relative to the mean corresponding values recorded after the same preincubation (absence or presence of oxidized LDL) and at the same concentration of acetylcholine in the rings from rats injected with the LCT emulsion. Mean values ( \pm SEM) refer to 30-34 rings, are ranged on a logarithmic scale, and are shown together with the statistical significance of differences between the two sets of data.

lower in the rings exposed to oxidized LDL than in those not exposed to oxidized LDL. This difference achieved statistical significance $(\mathrm{p} \leq 0.05)$ in the $10^{-8}$ to $10^{-4} \mathrm{M}$ range of acetylcholine concentration.

During the second relaxation test, the mean value for the fractional relaxation caused by acetylcholine was always higher in the STZ rats injected with the MCT:FO emulsion as compared to LCT emulsion. Such was the case both after incubation of the aortic rings in the absence or presence of oxidized LDL. Fig. 9 illustrates these findings after pooling of the data obtained under the latter two experimental conditions. The fractional relaxation caused by acetylcholine in the rings from rats injected with the MCT:FO emulsion ranged, relative to the mean corresponding value found at the same concentration of the cholinergic agent in aortic rings obtained from rats injected with the LCT emulsion and incubated under the same conditions (absence or presence of oxidized LDL), from $5.78 \pm 1.58(\mathrm{n}=30 ; \mathrm{p}<0.005)$ at $10^{-9} \mathrm{M}$ acetylcholine to $1.19 \pm 0.09(\mathrm{n}=30 ; \mathrm{p}>0.1)$ at $10^{-4} \mathrm{M}$ acetylcholine. In such a comparison, the SEM on the mean reference value (rats injected with the LCT emulsion) progressively decreased from 37.7 to $7.2 \%(n=34$ in both cases) as the concentration of acetylcholine was raised from $10^{-9}$ to $10^{-4} \mathrm{M}$.

Taken as a whole, these findings indicate that, in the STZ rats, the MCT:FO emulsion not only protects against the deleterious effects of oxidized LDL applied in vitro, but also improves aortic endothelial function in aortic rings not exposed in vitro to oxidized LDL. This suggests, that the MCT:FO emulsion opposed in vivo the functional alteration conceivably resulting from a diabetes-induced oxidative stress.

\section{Discussion}

The present findings reveal that prior injection of the MCT:FO emulsion to normal rats protects the vasomotor function of aortic rings against the deleterious action of oxidized LDL. Such is also the case in STZ rats, in which case the MCT:FO emulsion apparently even opposes in vivo a diabetes-induced alteration of aortic endothelial function. Such an alteration was already observed (8).

Three further considerations merit to be underlined. First, under the present experimental conditions, the intravenous injection of the MCT:FO emulsion results in a sustained enrichment of liver and, hence, presumably also aortic endothelial cells phospholipids in long-chain polyunsaturated $\omega 3$ fatty acids, indeed documented $20 \mathrm{~h}$ after such an injection (9).

Second, the intravenous injection of the MCT:FO emulsion to $\omega 3$-depleted rats was recently reported to result in a remodelling of ${ }^{86} \mathrm{Rb}^{+}$and ${ }^{45} \mathrm{Ca}^{2+}$ handling by aortic rings incubated in vitro (10).

Last, whether in control or $\omega 3$-depleted rats, the intravenous injection of the MCT:FO emulsion also improves cardiac function during in vitro aerobic reperfusion of ischemic hearts (11).

Taken as a whole, these converging findings support the view that the bolus intravenous injection of the MCT:FO emulsion represents a promising approach to cause a rapid and sustained enrichment of cell phospholipids in $\omega 3$ fatty acids and, by doing so, to protect or improve cardiovascular function.

\section{Acknowledgements}

This work was supported by a grant from the Région de Bruxelles-Capitale (convention BC-BR 246/2410, Brussels, Belgium) to Target Hit s.a. (Brussels, Belgium). We are grateful to C. Demesmaeker for secretarial help. The lipid emulsions were provided by B. Braun Melsungen (Melsungen, Germany).

\section{References}

1. Portois L, Deckelbaum RJ, Malaisse WJ and Carpentier YA: Accumulation rapide d'eicosapentaénoate dans les phospholipides cellulaires après injection intraveineuse d'une émulsion d'huile de poisson et de triglycerides à chaîne moyenne à des sujets normaux. Nutr Clin Metab 18 (Suppl 1): S53, 2004.

2. Portois L, Peltier S, Chardigny J-M, Sébédio J-L, Leverve X, Malaisse WJ and Carpentier YA: Correction of excessive triglyceride accumulation in the liver of $\omega$-3 fatty acid-depleted rats by a single i.v. injection of a medium-chain triglyceride:fish oil emulsion. Clin Nutr 24: 565, 2005.

3. Carpentier YA, Agiascogulu E, Oguzhan B, Zhang Y, Louchami K, Sener A, Portois L and Malaisse WJ: Modifications rapides des lipides et du métabolisme glucidique dans le muscle soleus de rats carencés en acides gras $\omega 3$ en réponse à l'injection intraveineuse d'une émulsion d'huile de poisson et de triglycérides à chaînes moyennes. Nutr Clin Metab 19 (Suppl 1): S66, 2005.

4. Havel RJ, Eder HA and Bragdon JH: The distribution and chemical composition of ultracentrifugally separated lipoproteins in human serum. J Clin Invest 34: 1345-1353, 1955.

5. Berkenboom G, Langer I, Carpentier Y, Grosfils K and Fontaine J: Ramipril prevents endothelial dysfunction induced by oxidized low-density lipoproteins: a bradykinin-dependent mechanism. Hypertension 30: 371-376, 1997. 
6. Fontaine D, Otto A, Fontaine J and Berkenboom G: Chronic HMG-CoA reductase inhibition and endothelial function of normocholesterolemic rat. Comparison with angiotensin converting enzyme inhibition. J Cardiovasc Pharmacol 40: 172-180, 2002.

7. Levy JV: Prostacyclin-induced contraction of isolated aortic strips from normal and spontaneously hypertensive rats (SHR). Prostaglandins 19: 517-525, 1980

8. Channon KM and Guzik TJ: Mechanisms of superoxide production in human blood vessels: relationship to endothelial dysfunction, clinical and genetic risk factors. J Physiol Pharmacol 53: 515-524, 2002.

9. Carpentier YA, Fontaine D, Otto A, Portois L, Fontaine $\mathrm{J}$ and Malaisse WJ: Sustained enrichment of liver phospholipids and triglycerides in eicosapentaenoate after a bolus intravenous injection of a medium-chain triglyceride:fish oil emulsion to streptozotocin (Type 1) and Goto-Kakizaki (Type 2) diabetic rats. Int J Mol Med 17: 643-647, 2006.
10. Courtois P, Louchami K, Portois L, Chardigny J-M, Sener A Carpentier YA and Malaisse WJ: Effects of a medium-chain triglyceride:fish oil emulsion administered intravenously to $\omega 3$ fatty acid-depleted rats on cationic fluxes in aortic rings. Int $\mathbf{J}$ Mol Med 16: 1089-1093, 2005.

11. Peltier S, Malaisse WJ, Portois L, Novel-Chaté V, Chardigny J-M, Carpentier YA and Leverve XM: L'administration in vivo d'une émulsion lipidique enrichie en huile de poisson améliore la fonction cardiaque post-ischémique de rats carencés en acides gras polyinsaturés n-3. Nutr Clin Metab 19 (Suppl 1): S66, 2005 . 